\title{
Evaluation of the limiting antigen avidity EIA (LAg) in people who inject drugs in Greece
}

\author{
G. K. NIKOLOPOULOS ${ }^{1,2,3 *}$, A. KATSOULIDOU ${ }^{4}$, M. KANTZANOU ${ }^{4}$, \\ C. ROKKA ${ }^{4}$, C. TSIARA ${ }^{3}$, V. SYPSA ${ }^{4}$, D. PARASKEVIS ${ }^{4}$, M. PSICHOGIOU $^{5}$, \\ S. FRIEDMAN ${ }^{6}$ AND A. HATZAKIS ${ }^{4}$ \\ ${ }^{1}$ Medical School, University of Cyprus, Nicosia, Cyprus \\ ${ }^{2}$ National Development and Research Institutes (NDRI), Transmission Reduction Intervention Project \\ (Athens site), New York, USA \\ ${ }^{3}$ Hellenic Centre for Disease Control and Prevention, Amarousio, Greece \\ ${ }^{4}$ Department of Hygiene, Epidemiology and Medical Statistics, Medical School, University of Athens, Athens, \\ Greece \\ ${ }^{5}$ First Department of Internal Medicine, Laiko General Hospital, University of Athens, Athens, Greece \\ ${ }^{6}$ National Development and Research Institutes (NDRI), New York, USA
}

Received 19 February 2016; Final revision 18 September 2016; Accepted 28 September 2016; first published online 26 October 2016

\section{SUMMARY}

This analysis assessed the utility of the limiting antigen avidity assay (LAg). Samples of people who inject drugs (PWID) in Greece with documented duration of HIV-1 infection were tested by LAg. A LAg-normalized optical density $(\mathrm{ODn}) \leqslant 1.5$ corresponds to a recency window period of 130 days. The proportion true recent (PTR) and proportion false recent (PFR) were estimated in 28 seroconverters and in 366 samples collected $>6$ months after HIV diagnosis, respectively. The association between LAg ODn and HIV RNA level was evaluated in 232 persons. The PTR was $85 \cdot 7 \%$. The PFR was $20 \cdot 8 \%$ but fell to $5 \cdot 9 \%$ in samples from treatment-naive individuals with long-standing infection ( $>1$ year), and to 0 in samples with the circulating recombinant form CRF35 AD. A LAg-based algorithm with a PFR of 3.3\% estimated a similar incidence trend to that calculated by analyses based on HIV-1 seroconversions. In recently infected persons indicated by LAg, the median $\log _{10}$ HIV RNA level was high (5·30, interquartile range $\left.4 \cdot 56-5 \cdot 90\right)$. LAg can help identify highly infectious HIV(+) individuals as it accurately identifies recent infections and is correlated with the HIV RNA level. It can also produce reliable estimates of HIV-1 incidence.

Key words: Drug injectors, HIV, incidence, LAg, recent infection.

\section{INTRODUCTION}

Monitoring of HIV incidence is essential for evaluating the effectiveness of interventions [1-4]. Nonetheless, epidemiological evidence on HIV consists mostly of

\footnotetext{
* Author for correspondence: Dr G. K. Nikolopoulos, Medical School, University of Cyprus, P.O. Box 20537, 1678 Nicosia, Cyprus.

(Email: gknikolopoulos@gmail.com)
}

prevalence data, reporting rates from surveillance systems, and estimates based on mathematical models with various assumptions. Prospective observational studies that produce estimates of HIV incidence are time-consuming and expensive, and may also suffer from bias [2-4].

If the number of people who are newly infected with HIV is known, HIV incidence can be calculated using cross-sectional approaches [5]. There have been substantial efforts to develop laboratory assays that can 
distinguish recent (e.g. acquired in the last 6 months) from long-standing HIV infections [3, 6]. The earliest application of less sensitive enzyme immunoassays (EIAs) has now been replaced by methods that capture HIV-specific IgG production (BED-CEIA) or measure the binding strength (avidity) between antibodies and viral antigens [3, 6-11]. The latter category includes the recently developed HIV-1 limiting antigen avidity EIA (LAg) [12-14].

Athens, Greece, experienced a large HIV-1 epidemic in people who inject drugs (PWID) between 2011 and 2013 [15-17]. In the context of interventions (ARISTOTLE programme and Transmission Reduction Intervention Project; TRIP) to respond to the epidemic [17, 18], many blood specimens from PWID with documented duration of HIV-1 infection were collected. This paper describes the performance characteristics of LAg in specimens of drug injectors, measures the correlation between normalized LAg optical density (ODn) and HIV RNA level, and compares LAg-based HIV-1 incidence estimates in PWID with those from prospective analyses based on HIV-1 seroconversions.

\section{METHODS}

\section{Subjects}

Samples were collected from participants of ARISTOTLE and of TRIP. ARISTOTLE [17] used a respondent-driven sampling design and took place between August 2012 and December 2013 as the main response to an HIV-1 outbreak in PWID that was recognized in early 2011 [15]. ARISTOTLE consisted of five successive rounds of HIV screening and recruited a total of 3320 PWID ( 1400 PWID were tested once in each round but were allowed to take part in the next waves of testing) whose characteristics are described in detail in [17]. HIV incidence estimations made use of all ARISTOTLE subjects while analyses on LAg performance and on its association with HIV RNA focused mainly on three subsets of ARISTOTLE participants: PWID with documented recent seroconversion; PWID with documented longterm infection; and PWID who participated in the first round only.

Given the longitudinal nature of ARISTOTLE, some PWID participated in multiple rounds of that intervention. Of people who were longitudinally observed in ARISTOTLE and were HIV negative at their first visit, 45 seroconverted during the course of the study. Of these, 28 were recent seroconverters defined as people who tested HIV positive in ARISTOTLE, had a previous negative test at most 6 months before the positive result in ARISTOTLE, and were antiretroviral treatment (ART) naive and without advanced HIV disease at the time their positive HIV sample was collected. Long-term infected persons were 209 ARISTOTLE participants who, at the time of blood sampling, had a documented (based on a previous positive test) HIV-1 infection $>6$ months ago. Of 366 available specimens for these persons, the duration of infection was between 6 and 12 months in 282 samples (77\%), and >1 year in 84 specimens $(23 \%)$.

TRIP, a multisite intervention that combines medical, laboratory, and social network tools to prevent HIV transmission [18], ran in Athens between 2013 and 2015. TRIP identifies people recently infected with HIV (defined as those who acquired HIV in the past 6 months) by using LAg (Sedia Biosciences Corporation, USA). TRIP and ARISTOTLE conducted LAg testing of seroconverters and long-term infected participants of ARISTOTLE, as defined previously, and HIV RNA testing of PWID who took part in the first round of ARISTOTLE. In 1404 drug injectors who participated in the first round of ARISTOTLE, 262 had detectable HIV RNA. Thirty of the 262 persons had started ART before joining ARISTOTLE and were excluded from further analyses on the association between LAg ODn and HIV RNA values.

Data on ART, CD4 T-cell counts at diagnosis, and on the diagnosis date of AIDS were obtained from the Greek HIV/AIDS registry and collaborating physicians. Data on HIV-1 subtypes and circulating recombinant forms (CRF) were retrieved from an existing database as described in [19].

\section{Laboratory methods}

Blood samples were tested by a microparticle anti-HIV1/2 EIA (AxSYM HIV-1/2 gO, Abbott Laboratories, USA) and confirmed by MP Diagnostics Western Blot (WB) (MP Biomedicals, USA). Specimens that were initially reactive $(\mathrm{S} / \mathrm{CO}, \geqslant 1 \cdot 0)$ or initially grey-zone reactive $(0 \cdot 9 \leqslant \mathrm{~S} / \mathrm{CO}<1 \cdot 0)$ in AxSYM HIV- $1 / 2 \mathrm{gO}$ were retested in duplicate. Results on WB p31 band reactivity were recorded. $\mathrm{p} 31$ can be detected $\sim 100$ days after infection and could be used as an indicator of recency in individuals with positive WB (Fiebig stage V: WB+/p31-) $[20,21]$. 
WB-confirmed samples were further tested by LAg. Samples with an ODn $\leqslant 2$ during the screening mode were subjected to confirmatory evaluation and tested in triplicate. If the median of the three ODn values was then $\leqslant 1 \cdot 5$, the seroconversion was classified as recent $(\mathrm{LAg}+)$. The ODn cut-off of 1.5 corresponds to a mean duration of recent infection (MDRI) of 130 days [14]. MDRI refers to the average time individuals spend in the biomarker-defined state of recent infection and is an important parameter of assays used in population-level estimation of HIV incidence.

HIV RNA was quantified in positive specimens obtained from participants of the first round of ARISTOTLE using Artus HI virus-1 RG RT-PCR (Qiagen, Germany).

\section{Validation of LAg and estimation of HIV-1 incidence}

The proportion true recent (PTR) and proportion false recent (PFR) of LAg along with their Wald or exact $95 \%$ confidence intervals (CIs) were calculated using samples of repeated testers in ARISTOTLE. PTR and PFR are also known and used in the literature as true recent rate (TRR) and false recent rate (FRR), respectively.

For incidence estimations, we used a LAg-based algorithm as described in guidelines developed by the World Health Organization (WHO) and the joint United Nations Programme (UNAIDS) on HIV [5] (Fig. 1). Our recent infection testing algorithm (RITA) reclassifies successively as non-recents samples with a LAg ODn $\leqslant 1.5$ if the participants had a documented HIV diagnosis $>1$ year ago, CD4 T-cell count $<200$ cells $/ \mu 1$ (around 3 months before or after sample collection), had developed AIDS within a month from sample collection or had started ART before their specimen was obtained in ARISTOTLE. For the first round of ARISTOTLE, as HIV RNA data were available, the algorithm was run twice excluding the ART-related information the second time but including HIV RNA data (reclassifying LAg-based recents as non-recents if their HIV RNA level was $\leqslant 1000$ copies $/ \mathrm{ml}$ ).

The MDRI used was that of LAg (130 days). The PFR of the applied RITA was the proportion of long-standing infections that were misclassified by the algorithm as recent and was calculated on the same population of drug injectors used for incidence estimations (surveyed in ARISTOTLE). PFR was estimated in HIV-positive samples with duration of infection $>6$ months, between 6 and 12 months, and $>1$ year. In addition, according to the WHO/UNAIDS guidance

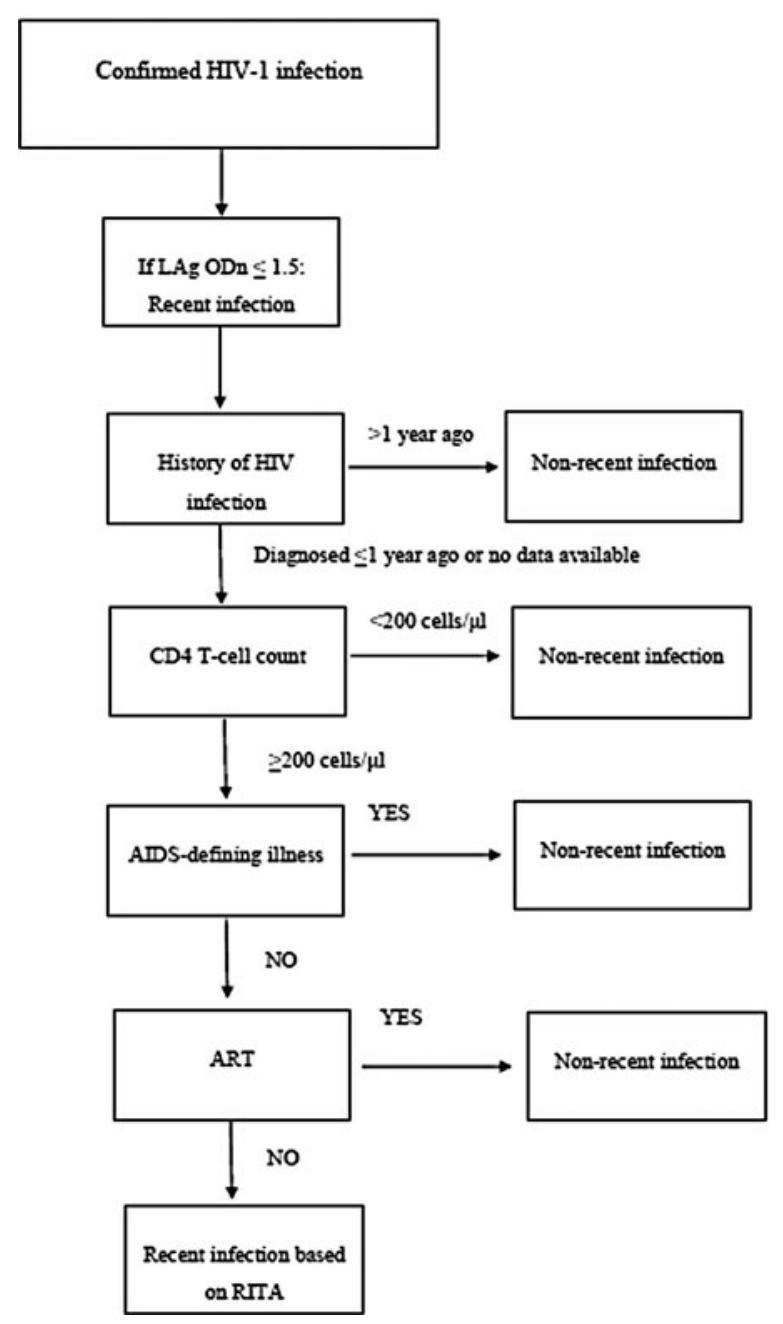

Fig. 1. Testing algorithm for recent infection based on the limiting antigen avidity (LAg) assay. ODn, normalized optical density; RITA, recent infection testing algorithm.

[5], a long-standing infection for incidence calculation has duration longer than twice the mean assay window period (for LAg: 130 days $\times 2=260$ days). Given that, PFR was also estimated in $213 \mathrm{HIV}$-positive samples with duration of infection $>260$ days and was the one used in incidence calculations.

The numbers of each round's positives and recents, and the PFR were entered on a web-based platform [Assay-Based Incidence Estimation (ABIE) version 2.0; http://www.incidence-estimation.org/page/cephiaoverview] that has been developed to facilitate the calculation of incidence estimates [22, 23].

\section{Statistical analysis}

Descriptive analyses included median values and interquartile ranges (IQRs). The assumption of normally 
distributed continuous variables was checked using the Shapiro-Wilk test. Mann-Whitney and KruskalWallis statistics were used to test the hypothesis that two or more statistical samples were drawn from the same populations. Spearman's rank correlation coefficient measured the statistical association between LAg ODn values and HIV RNA level. The $\chi^{2}$ and Fisher's exact tests were used to evaluate the independence of two categorical variables. Logistic regression models were used for dichotomous outcomes. HIV RNA levels were $\log _{10}$ transformed when used as a continuous variable. HIV RNA values also were grouped into six categories that correspond to $1 \log _{10}$ increment (i.e. $<100,\left[10^{2}-10^{3}\right),\left[10^{3}-10^{4}\right),\left[10^{4}-10^{5}\right)$, $\left[10^{5}-10^{6}\right), \geqslant 10^{6}$ copies $\left./ \mathrm{ml}\right)$.

All statistical analyses were conducted in Stata v. 12 (StataCorp., USA).

\section{Ethical statement}

The authors assert that all procedures contributing to this work comply with the ethical standards of the relevant national and institutional committees on human experiments and with the Helsinki Declaration of 1975, as revised in 2008. Each participant of ARISTOTLE and TRIP gave informed consent. Both studies were approved by Institutional Review Boards in Athens (Athens University Medical School and Hellenic Scientific Society for the study of AIDS and Sexually Transmitted Diseases, respectively). TRIP also obtained approval by the Institutional Review Board of the National Development and Research Institutes in New York City.

\section{RESULTS}

\section{Validation of LAg and of a LAg-based RITA (PTR and PFR)}

\section{PTR (sensitivity in individual diagnostics)}

The analysis on the PTR of LAg included 28 recent seroconverters (males, $85.7 \%$ ) with a median age of $35 \cdot 3$ years (IQR $31 \cdot 9-40 \cdot 2$ years).

LAg correctly identified $19 / 23$ cases $(82.6 \%, 95 \%$ CI 67.1-98.1) who had seroconverted during the last 130 days (the mean window period of $\mathrm{LAg}$ ) and 24/28 cases $(85.7 \%, 95 \%$ CI $72 \cdot 8-98 \cdot 7)$ who had become infected $<6$ months ago. When LAg results were combined with the reactivity of the WB p31 band, a p31-/ $\mathrm{LAg}+(\mathrm{ODn} \leqslant 1 \cdot 5)$ result correctly classified as recents $89 \cdot 3 \%$ (95\% CI $77 \cdot 8-100)$ of the seroconverters (25/28). The odds of being correctly classified as recent in the 6-month period between the last negative and the first positive test were not affected by the exact time elapsed from the last negative to the first positive test (odds ratio $1 \cdot 00,95 \%$ CI $0 \cdot 97-1 \cdot 03$ ).

Of the 28 seroconverters, 13 were classified as CRF14 BG (46.4\%), seven as subtype B (25\%), two as CRF35 $\mathrm{AD}(7 \cdot 1 \%)$, and two as recombinants of the previous groups $(7 \cdot 1 \%)$. The four false recents were identified in CRF14 BG (two), subtype B (one), and in the recombinants (one). The estimated PTR across molecular groups were: CRF14 BG (11/ $13,84 \cdot 6 \%)(95 \%$ CI $54 \cdot 6-98 \cdot 1)$; CRF35 AD $(2 / 2$, $100 \%)$; subtype B $(6 / 7,85 \cdot 7 \%)(95 \%$ CI $42 \cdot 1-99 \cdot 6)$; and recombinants $(1 / 2,50 \%)(95 \%$ CI $1 \cdot 3-98 \cdot 7)$. There was not any statistically significant difference of PTR in the molecular groups $(P=0 \cdot 66)$.

\section{PFR (1 - specificity in individual diagnostics)}

The analysis on LAg PFR included 209 ARISTOTLE participants (males, $78.5 \%$ ) with long-term infection and a median age of $34 \cdot 1$ years (IQR $30 \cdot 2-38 \cdot 9$ years) at the time of blood sampling. In total, 366 specimens were available from these participants.

LAg alone (prior to RITA adjustments) misclassified 76/366 specimens (PFR 20.8\%, 95\% CI 16.624.9 ) as recent infections (Table 1). Of those who had already received ART before sample collection, the PFR was substantial (around 35\%) regardless of the duration of HIV-1 infection. By contrast, the PFR in ART-naive participants was $11.5 \%(95 \% \mathrm{CI}$ $7 \cdot 2-15 \cdot 7)$ overall and fell to $5 \cdot 9 \%(95 \%$ CI $0-12 \cdot 3)$ when the samples had been obtained at least 1 year after infection. Using data on AIDS and CD4 T-cell counts further improved the PFR estimates. In particular, the overall RITA-based PFR was 4.9\% (95\% CI 2.7-7.1) and it became zero in samples collected from HIV-1-infected individuals who had been infected more than 1 year ago (Table 1). All estimates remained unchanged when one sample per participant was analysed (either the sample with the minimum duration or the sample with the maximum duration of HIV-1 infection).

The results were also similar on samples that had been collected more than twice the mean LAg recency period $(2 \times 130=260$ days $)$ after infection. Combining available clinical and laboratory information (CD4 Tcell count, HIV and AIDS diagnosis dates, and ART) for 213 samples of 150 participants, the RITA 
Table 1. Results on LAg PFRs using 366 samples from 209 participants of the ARISTOTLE programme in Athens, Greece with documented seroconversion at least 6 months before the date their sample for PFR analyses was collected

\begin{tabular}{|c|c|c|c|}
\hline Time between blood collection and documented seroconversion & $N$ & $\mathrm{LAg} \leqslant 1 \cdot 5$ & $\mathrm{PFR} \%(95 \% \mathrm{CI})$ \\
\hline \multicolumn{4}{|l|}{ All } \\
\hline$>6$ months & 366 & 76 & $20 \cdot 8(16 \cdot 6-24 \cdot 9)$ \\
\hline 6-12 months & 282 & 61 & $21 \cdot 6(16 \cdot 8-26 \cdot 4)$ \\
\hline$>260$ days* & 213 & 40 & $18 \cdot 8(13 \cdot 5-24 \cdot 0)$ \\
\hline$>1$ year & 84 & 15 & $17 \cdot 9(9 \cdot 7-26 \cdot 1)$ \\
\hline \multicolumn{4}{|l|}{ ART before blood collection } \\
\hline$>6$ months & 148 & 51 & $34 \cdot 5(26 \cdot 8-42 \cdot 1)$ \\
\hline 6-12 months & 115 & 39 & $33 \cdot 9(25 \cdot 3-42 \cdot 6)$ \\
\hline$>260$ days & 88 & 30 & $34 \cdot 1(24 \cdot 2-44 \cdot 0)$ \\
\hline$>1$ year & 33 & 12 & $36 \cdot 4(19 \cdot 9-52 \cdot 8)$ \\
\hline \multicolumn{4}{|l|}{ ART-naive at blood collection } \\
\hline$>6$ months & 218 & 25 & $11 \cdot 5(7 \cdot 2-15 \cdot 7)$ \\
\hline $6-12$ months & 167 & 22 & $13 \cdot 2(8 \cdot 0-18 \cdot 3)$ \\
\hline$>260$ days & 125 & 10 & $8 \cdot 0(3 \cdot 2-12 \cdot 8)$ \\
\hline$>1$ year & 51 & 3 & $5 \cdot 9(0-12 \cdot 3)$ \\
\hline \multicolumn{4}{|l|}{ RITA algorithm $\dagger$} \\
\hline$>6$ months & 366 & 18 & $4 \cdot 9(2 \cdot 7-7 \cdot 1)$ \\
\hline $6-12$ months & 282 & 18 & $6 \cdot 4(3 \cdot 5-9 \cdot 2)$ \\
\hline$>260$ days & 213 & 7 & $3 \cdot 3(0 \cdot 9-5 \cdot 7)$ \\
\hline$>1$ year & 84 & 0 & 0 \\
\hline
\end{tabular}

ART, Antiretroviral treatment; CI, Wald-based confidence intervals; LAg, limiting antigen avidity assay; PFR, proportion false recent; RITA, recent infection testing algorithm.

* 260 days is twice the recency window of LAg (130 days). It was used to define long-standing infections in calculations of PFR for incidence estimation.

$\dagger$ Recent infection testing algorithm (RITA): positives who had LAg normalized optical density (ODn) $\leqslant 1.5$ are reclassified successively as non-recents based on clinical and laboratory data (HIV diagnosis $>1$ year ago, CD4 T-cell count $<200$ cells $/ \mu 1$ 3 months before or after blood collection, diagnosis of AIDS and receipt of antiretroviral treatment before sampling).

misclassified seven samples as recents (PFR 3.3\%, 95\% CI 0.9-5.7). The estimated PFR did not change when the analysis focused on single samples per participant [either the earliest participant's sample with the minimum duration of HIV-1 infection (59 samples in total for PFR estimation: $3 \cdot 4 \%, 95 \%$ CI $0-8 \cdot 0$ ) or the latest sample with the maximum duration of HIV-1 infection (150 samples in total for PFR estimation: $3 \cdot 3 \%, 95 \%$ CI $0 \cdot 5-6 \cdot 2)$ ].

Data on HIV-1 subtypes and CRF were available for 133 samples $(36 \cdot 4 \%)$. Of these, 127 samples had a duration of HIV-1 infection $>6$ months but $<12$ months. The major groups were CRF14 BG (62), subtype B (23), CRF35 AD (22), and subtype A (12). The PFRs were high in CRF14 BG [17/62 (27.4\%) false recents, 95\% CI 16.9-40.2], and in subtypes A $(3 / 12,25 \% ; 95 \%$ CI $5 \cdot 5 \%-57 \cdot 2 \%)$ and B (7/ $23,30 \cdot 4 \%$; $95 \%$ CI $13 \cdot 2-52 \cdot 9)$. The PFR was lower in recombinants of the four major groups $(1 / 14$, $7 \cdot 1 \% ; 95 \%$ CI $0 \cdot 2-33.9)$ and became zero in CRF35 AD.

\section{LAg ODn values and HIV RNA level}

In 232 ART-naive HIV-1 positives of round 1 in ARISTOTLE with detectable HIV RNA, LAg ODn was $\leqslant 1.5(\mathrm{LAg}+$, i.e. indicating a recent infection) in 57 individuals $(24 \cdot 6 \%)$ while the WB p31 band was non-reactive (p31-, i.e. indicating an infection $<100$ days ago) in 31 participants $(13 \cdot 4 \%)$. Combining $\mathrm{LAg}$ and $\mathrm{p} 31$ results, 11 individuals $(4 \cdot 7 \%)$ were indicated as recently infected by both assays (LAg+ and p31-) while 66 persons (28.5\%) were classified as recents by only one of these tests (LAg+ but p31+ or LAg- but p31-).

Table 2 shows HIV RNA levels of all participants and across various subgroups $(A-G)$. Significantly $(P=0.06)$ more participants in group $\mathrm{C}$ with $\mathrm{LAg}$ ODn $\leqslant 1 \cdot 5(\mathrm{LAg}+)$ had a viral load of $\geqslant 10^{6}$ copies/ $\mathrm{ml}$ than did those in group $\mathrm{G}$ with LAg ODn $>1.5$ (LAg-). Almost half $(45 \cdot 5 \%)$ of the individuals in group A that included recents indicated by both assays (p31-/LAg+) had HIV RNA levels $\geqslant 10^{6}$ copies $/ \mathrm{ml}$. 
Table 2. Median $\log _{10}$ HIV RNA values (copies/ml) and the distribution of HIV RNA levels by recency status in 232 antiretroviral treatment-naive participants of the first round of the ARISTOTLE programme in Athens, Greece

\begin{tabular}{|c|c|c|c|c|c|c|}
\hline Recency status* & $N$ & $\begin{array}{l}\text { Median } \log _{10} \text { HIV } \\
\text { RNA (IQR) }\end{array}$ & HIV RNA $\geqslant 10^{6}$ & HIV RNA $\left[10^{5}-10^{6}\right)$ & HIV RNA $\left[10^{4}-10^{5}\right)$ & HIV RNA $<10^{4}$ \\
\hline All (irrespective of recency status) & 232 & $5 \cdot 11(4 \cdot 44-5 \cdot 52)$ & $22(9 \cdot 5 \%)$ & $102(43 \cdot 9 \%)$ & $76(32 \cdot 8 \%)$ & $32(13 \cdot 8 \%)$ \\
\hline $\begin{array}{l}\text { (A) Recent infection indicated by both assays } \dagger \\
\text { (p31- and } \mathrm{LAg}+\text { ) }\end{array}$ & 11 & $5 \cdot 64(5 \cdot 08-6 \cdot 13)$ & $5(45 \cdot 5 \%)$ & $4(36 \cdot 3 \%)$ & $2(18 \cdot 2 \%)$ & $0(0 \%)$ \\
\hline $\begin{array}{l}\text { (B) Recent infection indicated by LAg but not } \\
\text { by p31 (p31+ and LAg+) }\end{array}$ & 46 & $5 \cdot 23(4 \cdot 33-5 \cdot 70)$ & $5(10 \cdot 9 \%)$ & $23(50 \cdot 0 \%)$ & $12(26 \cdot 1 \%)$ & $6(13 \cdot 0 \%)$ \\
\hline (C) Recent infection indicated by $\mathrm{LAg}(\mathrm{LAg}+)$ & 57 & $5 \cdot 30(4 \cdot 56-5 \cdot 90)$ & $10(17 \cdot 5 \%)$ & $27(47 \cdot 4 \%)$ & $14(24 \cdot 6 \%)$ & $6(10 \cdot 5 \%)$ \\
\hline $\begin{array}{l}\text { (D) Recent infection indicated by one assay } \\
\text { (p31- but LAg- or LAg+ but p31+) }\end{array}$ & 66 & $5 \cdot 30(4 \cdot 53-5 \cdot 70)$ & $8(12 \cdot 1 \%)$ & $36(54 \cdot 6 \%)$ & $16(24 \cdot 2 \%)$ & $6(9 \cdot 1 \%)$ \\
\hline $\begin{array}{l}\text { (E) Long-term infection indicated by both } \\
\text { assays (p31+ and LAg-) }\end{array}$ & 155 & $4 \cdot 90(4 \cdot 40-5 \cdot 39)$ & $9(5 \cdot 8 \%)$ & $62(40 \%)$ & $58(37 \cdot 4 \%)$ & $26(16 \cdot 8 \%)$ \\
\hline $\begin{array}{l}\text { (F) Long-term infection indicated by LAg but } \\
\text { not by p31 (p31- and LAg-) }\end{array}$ & 20 & $5 \cdot 49(5 \cdot 08-5 \cdot 71)$ & $3(15 \%)$ & $13(65 \%)$ & $4(20 \%)$ & $0(0 \%)$ \\
\hline $\begin{array}{l}\text { (G) Long-term infection indicated by LAg } \\
\text { (LAg-) }\end{array}$ & 175 & $4.99(4 \cdot 44-5 \cdot 43)$ & $12(6 \cdot 9 \%)$ & $75(42 \cdot 9 \%)$ & $62(35 \cdot 4 \%)$ & $26(14 \cdot 8 \%)$ \\
\hline
\end{tabular}

LAg, Limiting antigen avidity assay; IQR, interquartile range.

Comparisons between groups that do not overlap:

HIV RNA as continuous variable: A vs. B $(P=0 \cdot 07)$; E vs. F $(P=0 \cdot 002)$; C vs. $\mathrm{G}(P=0 \cdot 02)$.

HIV RNA as categorical variable: A vs. B $(P=0 \cdot 04)$; E vs. F $(P=0 \cdot 02)$; $\mathrm{C} v s . \mathrm{G}(P=0 \cdot 06)$

* The groups overlap. The sum of all frequencies in the second column does not equal the total number of subjects (232) in these analyses. Groups A and B are subsets of group $\mathrm{C}$; groups $\mathrm{E}$ and $\mathrm{F}$ are subsets of group $\mathrm{G}$; group $\mathrm{D}$ includes individuals of groups $\mathrm{C}$ and $\mathrm{G}$; the sum of frequencies of groups $\mathrm{C}$ and $\mathrm{G}$ equals total $N$.

$\dagger$ Assays include the LAg and the Western Blot (WB) p31. An infection was classified as recent if the LAg normalized optical density score was $\leqslant 1 \cdot 5$ (LAg+) or the p31 band of the WB-confirmed HIV-1 infection was negative (p31-), respectively. 
This number is significantly higher $(P=0 \cdot 04)$ than the corresponding percentage (10.9\%) in the individuals of group B who were classified as recents by LAg but not by $\mathrm{p} 31$.

The median $\log _{10}$ HIV RNA level of the 232 ARTnaive HIV-1 positive participants in the first round of ARISTOTLE was $5 \cdot 11$ (IQR 4.44-5.52). The Spearman coefficient of correlation between LAg ODn and $\log _{10}$ HIV RNA level was $-0 \cdot 17(P=$ $0 \cdot 01)$. Viral load was significantly higher in recently (group $\mathrm{C}$ with $\mathrm{LAg}$ ODn $\leqslant 1.5$ ) infected persons $(5 \cdot 30, P=0 \cdot 02)$ than in long-term (group $\mathrm{G})$ infectees (4.99). The median viral load peaked in individuals in group A who were indicated as recents by both LAg and the WB p31 band (5.64, $P=0.07$ compared to individuals of group B who were classified as recents by LAg but not by p31). Interestingly, the median viral load in LAg-based long-standing infections with p31 negativity (group F: 5.49 ) was significantly higher $(P=0.002)$ than in long-term infectees (group E), as indicated by both assays (4.90).

\section{Calculation of HIV-1 incidence}

As shown in Table 3, HIV-1 prevalence fell from $19 \cdot 3 \%$ in the first round of ARISTOTLE to $14.5 \%$ over the last recruitment period. By combining LAg data with laboratory and clinical information (RITA), we found that the proportion of recents decreased over the course of the project from $20 \cdot 3 \%$ $(n=55)$ to around $7.9 \%(n=16)$. Using the previously calculated PFR of $3 \cdot 3 \%$, the RITA-based estimate of HIV-1 incidence showed a clear decreasing trend from $12.62 \%(95 \%$ CI $8 \cdot 38-16 \cdot 86)$ in the first round to $2 \cdot 41 \%(0 \cdot 14-4 \cdot 67)$ at the end of the study. This corresponds to an incidence reduction of $\sim 81 \%$, which is very similar to the estimated incidence decrease $(78 \%)$ calculated on seroconverters in repeated testers in ARISTOTLE [24].

When HIV RNA replaced ART in the algorithm applied to the data of the first round of ARISTOTLE, the RITA-based number of recents became $59(21 \cdot 8 \%$ of HIV positives) and the estimated HIV incidence slightly increased to $13 \cdot 71 \%$. However, its $95 \%$ CI (9.31-18.11) substantially overlaps with the ARTbased CI given above $(8 \cdot 38-16 \cdot 86)$.

\section{DISCUSSION}

Approaches based on cross-sectional biomarkers including the LAg [12-14] could be reliable alternatives to lengthy and costly follow-up studies for calculating HIV incidence [3, 6-8]. In samples of HIV-infected PWID collected in Greece during an HIV-1 outbreak, the ability of LAg alone to accurately identify HIV-1 infections with a duration of $<6$ months was around $85 \%$. In addition, LAg was significantly correlated with HIV RNA values, which are associated with HIV transmission. In the absence of ART and of advanced disease, the PFR of LAg drops from $20 \%$ to $5 \%$, and becomes zero for infections with at least 1 year's duration. An algorithm based on LAg and clinical information, and using the LAg window of recency (130 days) and an estimated PFR of $3 \cdot 3 \%$, detected a similar incidence trend to that based on seroconverters. Replacing ART with HIV RNA in RITA applied to a subset of the data did not substantially change the incidence estimate and its $95 \%$ CI.

The maturation of serological response is influenced by several factors including individual and population variability in immune reaction, and subtype bias liability [3, 25]. Population variation was probably limited in this study because the analyses focused only on people who use drugs. However, HIV-1 subtypes influence the kinetics of antibody maturation. The BED-CEIA assay, for example, has showed varying performance across populations with different HIV subtypes and seems to overestimate incidence [3, 26-30]. To overcome subtype effects, LAg contains a recombinant protein derived from the immunodominant region of glycoprotein 41, from multiple subtypes of the HIV-1 group $\mathrm{M}[12,13]$. However, the performance of LAg may also vary across different HIV-1 subtypes, with higher PFRs being observed in people infected with subtype D [30-35]. Infections with subtype D could be associated with weak antibody responses that persist over time and this may affect LAg results [30, 36]. The most frequently detected subtypes in drug injectors in Greece during the recent epidemic include two recombinants (CRF14 BG, CRF35 AD), and subtypes A and $B$ [15]. The PTR was not affected by the circulating subtypes but the analysis was based on a rather small sample $(n=28)$. The PFR was high $(>20 \%)$ in subtypes $\mathrm{A}$ and $\mathrm{B}$, and in CRF14 BG but fell to $7 \cdot 7 \%$ in a group of recombinants of the four major strains and became 0 in CRF35 AD sequences. However, the estimation of PFR across subtypes and CRFs suffers from small sample sizes (available data for $36 \%$ of specimens collected $>6$ months after infection).

HIV incidence assays may falsely identify long-term infections as recent when blood samples are collected from elite controllers, people on ART, and those with 
Table 3. Estimates of HIV-1 incidence in the five rounds of the ARISTOTLE programme in Athens, Greece using LAg, clinical and laboratory information in the context of a RITA* method

\begin{tabular}{|c|c|c|c|c|c|}
\hline Period & $\begin{array}{l}\text { Round } 1 \\
20 \text { Aug. 2012-3 Dec. } \\
2012\end{array}$ & $\begin{array}{l}\text { Round } 2 \\
4 \text { Dec. 2012-18 Mar. } \\
2013\end{array}$ & $\begin{array}{l}\text { Round } 3 \\
19 \text { Mar. 2013-16 June } \\
2013\end{array}$ & $\begin{array}{l}\text { Round } 4 \\
17 \text { June } 2013-18 \\
\text { Sep. } 2013\end{array}$ & $\begin{array}{l}\text { Round } 5 \\
19 \text { Sep. 2013-16 Dec } \\
2013\end{array}$ \\
\hline No. tested $\dagger$ & 1401 & 1438 & 1429 & 1406 & 1401 \\
\hline Negatives & 1130 & 1201 & 1182 & 1188 & 1198 \\
\hline Positives $(\%)$ & $271(19 \cdot 3 \%)$ & $237(16 \cdot 5 \%)$ & $247(17 \cdot 3 \%)$ & $218(15 \cdot 5 \%)$ & $203(14 \cdot 5 \%)$ \\
\hline $\begin{array}{l}\text { Recents ( } \% \text { of positives) based on } \\
\text { LAg ODn } \leqslant 1 \cdot 5\end{array}$ & $72(26 \cdot 6 \%)$ & $60(25 \cdot 3 \%)$ & $63(25 \cdot 5 \%)$ & $63(28 \cdot 9 \%)$ & $43(21 \cdot 2 \%)$ \\
\hline RITA-based recents ( $\%$ of positives) & $55(20 \cdot 3 \%)$ & $41(17 \cdot 3 \%)$ & $31(12 \cdot 6 \%)$ & $30(13 \cdot 8 \%)$ & $16(7 \cdot 9 \%)$ \\
\hline Incidence per 100 p-yr estimated by RITA & $12 \cdot 62 \%(8 \cdot 38-16 \cdot 86)$ & $8 \cdot 55 \%(5 \cdot 15-11 \cdot 96)$ & $5 \cdot 99 \%(2 \cdot 91-9 \cdot 06)$ & $5 \cdot 94 \%(2 \cdot 99-8 \cdot 90)$ & $2 \cdot 41 \%(0 \cdot 14-4 \cdot 67)$ \\
\hline $\begin{array}{l}\text { Incidence per } 100 \mathrm{p} \text {-yr estimated by } \\
\text { seroconverters among repeated testers in } \\
\text { ARISTOTLE }\end{array}$ & $7 \cdot 76 \%(4 \cdot 60-13 \cdot 11)$ & $5 \cdot 88 \%(3 \cdot 70-9 \cdot 33)$ & \multicolumn{2}{|c|}{$2 \cdot 91 \%(1 \cdot 57-5 \cdot 41) \S$} & $1 \cdot 71 \%(0 \cdot 55-5 \cdot 31) \|$ \\
\hline
\end{tabular}

LAg, Limiting antigen avidity assay; ODn, normalized optical density; p-yr, person-years; RITA, recent infection testing algorithm.

* Positives who had normalized LAg ODn $\leqslant 1.5$ are reclassified successively based on clinical and laboratory data (HIV diagnosis $>1$ year ago, CD4 T-cell count $<200$ cell/ $\mu 1$ 3 months before or after blood collection, diagnosis of AIDS and receipt of antiretroviral treatment before sampling).

$\dagger$ Numbers for LAg-based incidence analyses.

† Period: December 2012-April 2013.

§ Period: April 2013-August 2013

|| Period: August 2013-December 2013. 
advanced disease [25, 28, 37]. In our analyses, the PFR of LAg alone was high $(35 \%)$ when measured on infected individuals receiving ART. Similar figures $(58.8 \%)$ have also been reported by other researchers [34]. However, PFR on specimens that had been collected at least 1 year ago became even zero when a RITA algorithm was used that included data on ART, AIDS diagnosis date, and CD4 T-cell counts. A limitation of this RITA-based analysis is the relatively small sample size that was used for PFR estimations but, on the other hand, as recommended, PFR was measured on the same population for which incidence calculations were made [5]. Another limitation is that the mean recency window period of the LAg-based algorithm was not calculated as only a few samples from 45 seroconverters ( 28 with documented $<6$ months duration of infection) were available; therefore, the analyses were based on the mean recency window period of LAg alone (130 days) as reported in the literature [14].

A technical report by WHO and UNAIDS stated that LAg has lower PFR and leads to more accurate estimates of HIV incidence than other methods [25]. $\mathrm{LAg}$ had indeed showed a lower misclassification frequency than BED in people with AIDS in its initial evaluation [12]. Studies in Uganda, other African countries, Germany, Brazil, and the United States produced similar findings $[30,33,34,37-40]$. However, LAg alone has still some limitations and given the weaknesses of all single assays, further developments and evaluations are necessary including assessing the performance of many biomarker-based assays used in combination [23, 34]. Some multiassay or multi-analyte algorithms including LAg and other avidity measurements have already been tested with very encouraging results [28, 33, 38, 41-44].

The estimated incidence of HIV-1 infection that was assessed longitudinally in ARISTOTLE based on 45 seroconversions [24] was compared to the incidence estimated from cross-sectional samples (RITA method) collected in each of the five rounds of ARISTOTLE. The RITA approach indicated, in general, higher infection rates for each time period than the traditional, longitudinal approach. However, the $95 \%$ CIs of the estimates produced by the two methods substantially overlap. In addition, the RITA-based estimates refer to the 4- to 6-month period before the cross-sectional data collection, i.e. to the mean recency window of LAg. This means that each RITA-based estimate should be compared to the seroconverters-based incidence of the previous time period. In this case, the point estimates of the two methods become similar. For instance, the RITA-based point estimate of incidence in the fifth round of ARISTOTLE $(2 \cdot 41 \%)$ is much closer to the seroconverters-based estimate of the fourth round $(2 \cdot 91 \%)$. It should also be noted that the estimated reductions of incidence between August 2012 and December 2013 produced by the two methods were almost identical: decrease based on incidence estimates from HIV seroconversions in repeated testers $=78 \%$ vs. decrease from RITA-based estimates $=81 \%$. The reduction of HIV-1 incidence in drug injectors was also reflected in Greek surveillance data with reported diagnoses starting to decrease in June of 2013, following the RITA-based estimated decline in HIV-1 incidence during the first months of 2013 [45]. A LAg-based approach was also able to reliably detect incidence trends in young individuals in an African setting [46].

In the round 1 of ARISTOTLE, the applied RITA was rerun substituting ART with viral load. Being the last step of the algorithm, HIV RNA ( $\leqslant 1000$ copies/ $\mathrm{ml}$ ) reclassified four individuals fewer than ART did as non-recents. This resulted in a slightly increased HIV incidence but the two CIs substantially overlap. Given the limitations of our work, it seems that if administrative information on the date of ART start is accurate, ART can be safely used in RITA-based estimations instead of or along with HIV RNA.

Although the sample of seroconverters in this study was small $(n=28)$, the analysis demonstrated a good ability $($ PTR $=85 \%)$ of $\mathrm{LAg}$ to identify samples from persons who had acquired HIV $<6$ months ago. Our estimate is substantially better than those reported by Konikoff et al. (43\%) [38] and the German seroconverter cohort (48-63\%) [30]. The PTR further improved reaching $89 \%$ when it was combined with WB data (p31 reactivity).

LAg ODn values were significantly correlated with HIV RNA levels. In particular, half of the WBconfirmed HIV-1-infected individuals with LAg ODn $\leqslant 1.5$ and without WB $\mathrm{p} 31$ reactivity had very high HIV RNA concentrations ( $>10^{6}$ copies $\left./ \mathrm{ml}\right)$, typical of acute infection [47]. HIV RNA values comprise a good indicator of HIV transmissibility [48]. Yet, the cost of HIV RNA assays remains high while the elevated transmission risk during early infection is not explained by viral load values alone [48, 49]. Given the acceptable PTR, the strong correlation of LAg values with HIV RNA levels, LAg use alone or in combination with information provided by WB p31 in settings where WB remains, as a confirmatory 
test, in diagnostic algorithms, could be cheaper than HIV RNA testing and a reliable tool in social network interventions like TRIP [18] that aim to reduce the spread of HIV by recruiting people at the initial phase of increased transmissibility.

In conclusion, a LAg-based RITA had relatively low PFRs in a population of drug injectors and can be used in serial cross-sectional surveys to reliably monitor time trends in HIV incidence. LAg, in conjunction with testing histories and WB p31 results in settings where they are available, could also be a reliable and relatively cheap tool in resource-limited interventions targeting highly infectious persons [18]. Of note, $\mathrm{LAg}$ also performs well with dried blood specimens [50] while a rapid LAg has been developed with promising characteristics [51]. Since these types of LAg are less dependent on skilled staff and complicated procedures, they can facilitate LAg-based interventions and incidence measurements in the field.

\section{ACKNOWLEDGEMENTS}

Support for ARISTOTLE and several authors was provided by EU NSRF 2007-2013 that was co-funded by the European Social Fund and Greek national resources. Additional financial support was provided by the Hellenic Scientific Society for the study of AIDS and Sexually Transmitted Diseases. We also acknowledge support from National Institute on Drug Abuse (NIDA) (grants P30 DA11041-Center for Drug Use and HIV Research; and DP1 DA034989 Preventing HIV Transmission by Recently-Infected Drug Users). The International AIDS Society and the National Institute on Drug Abuse (NIDA) have supported the post-doc fellowship of the first author. The United States Centers for Disease Control have provided some of the LAg kits used in the analyses.

The content is solely the responsibility of the authors and does not necessarily represent the official views of the National Institutes of Health or other funding agencies.

\section{DECLARATION OF INTEREST}

None.

\section{REFERENCES}

1. Lasry A, et al. HIV sexual transmission risk among serodiscordant couples: assessing the effects of combining prevention strategies. AIDS 2014; 28: 1521-1529.
2. Mastro TD, et al. Estimating HIV incidence in populations using tests for recent infection: issues, challenges and the way forward. Journal of HIVIAIDS Surveillance \& Epidemiology 2010; 2: 1-14.

3. Mastro TD. Determining HIV incidence in populations: moving in the right direction. Journal of Infectious Diseases 2013; 207: 204-206.

4. Brookmeyer R. Measuring the HIV/AIDS epidemic: approaches and challenges. Epidemiologic Reviews 2010; 32: 26-37.

5. WHO/UNAIDS. When and how to use assays for recent infection to estimate HIV incidence at population level. Geneva, Switzerland, 2011.

6. Murphy G, Parry J V. Assays for the detection of recent infections with human immunodeficiency virus type 1 . Eurosurveillance 2008; 13: pii:18966.

7. Parekh BS, et al. Quantitative detection of increasing HIV type 1 antibodies after seroconversion: a simple assay for detecting recent HIV infection and estimating incidence. AIDS Research and Human Retroviruses 2002; 18: 295-307.

8. Dobbs T, et al. Performance characteristics of the immunoglobulin G-capture BED-enzyme immunoassay, an assay to detect recent human immunodeficiency virus type 1 seroconversion. Journal of Clinical Microbiology 2004; 42: 2623-2628.

9. Young CL, et al. Evaluation of a sensitive/less sensitive testing algorithm using the bioMérieux Vironostika-LS assay for detecting recent HIV-1 subtype B' or E infection in Thailand. AIDS Research and Human Retroviruses 2003; 19: 481-486.

10. Hall HI, et al. Estimation of HIV incidence in the United States. Journal of the American Medical Association 2008; 300: 520-529.

11. Suligoi B, et al. Identifying recent HIV infections using the avidity index and an automated enzyme immunoassay. Journal of Acquired Immune Deficiency Syndromes 2003; 32: 424-428.

12. Duong YT, et al. Detection of recent HIV-1 infection using a new limiting-antigen avidity assay: potential for HIV-1 incidence estimates and avidity maturation studies. PLoS ONE 2012; 7: e33328.

13. Wei $\mathbf{X}$, et al. Development of two avidity-based assays to detect recent HIV type 1 seroconversion using a multisubtype gp41 recombinant protein. AIDS Research and Human Retroviruses 2010; 26: 61-71.

14. Duong YT, et al. Recalibration of the limiting antigen avidity EIA to determine mean duration of recent infection in divergent HIV-1 subtypes. PLOS ONE 2015; 10: e0114947.

15. Paraskevis D, et al. Economic recession and emergence of an HIV-1 outbreak among drug injectors in athens metropolitan area: a longitudinal study. PLOS ONE 2013; 8: e78941.

16. Nikolopoulos GK, et al. Big Events in Greece and HIV infection among people who inject drugs. Substance Use \& Misuse 2015; 50: 825-838.

17. Hatzakis A, et al. Design and baseline findings of a large-scale rapid response to an HIV outbreak in people 
who inject drugs in Athens, Greece: the ARISTOTLE programme. Addiction 2015; 110: 1453-1467.

18. Friedman SR, et al. Socially-integrated transdisciplinary HIV prevention. AIDS and Behavior 2014; 18: 18211834.

19. Paraskevis D, et al. Enhanced HIV-1 surveillance using molecular epidemiology to study and monitor HIV-1 outbreaks among intravenous drug users (IDUs) in Athens and Bucharest. Infection, Genetics and Evolution 2015; 35: 109-121.

20. Fiebig EW, et al. Dynamics of HIV viremia and antibody seroconversion in plasma donors: implications for diagnosis and staging of primary HIV infection. AIDS 2003; 17: 1871-1879.

21. McMichael AJ, et al. The immune response during acute HIV-1 infection: clues for vaccine development. Nature Reviews Immunology 2010; 10: 11-23.

22. Kassanjee R, et al. A new general biomarker-based incidence estimator. Epidemiology 2012; 23: 721-728.

23. Kassanjee R, McWalter TA, Welte A. Short Communication: Defining optimality of a test for recent infection for HIV incidence surveillance. AIDS Research and Human Retroviruses 2014; 30: 45-49.

24. Sypsa V, et al. Trends in HIV-1 incidence during an outbreak among injecting drug users in Athens, Greece: results of a serobehavioral survey (ARISTOTLE program) [Abstract]. 20th International AIDS Conference, 2014.

25. WHO/UNAIDS. Technical Update on HIV incidence assays for surveillance and epidemic monitoring. Geneva, Switzerland: 2013.

26. Karita E, et al. Investigating the utility of the HIV-1 BED capture enzyme immunoassay using cross-sectional and longitudinal seroconverter specimens from Africa. AIDS 2007; 21: 403-408.

27. Guy R, et al. Accuracy of serological assays for detection of recent infection with HIV and estimation of population incidence: a systematic review. Lancet Infectious Diseases 2009; 9: 747-759.

28. Brookmeyer R, et al. Cross-sectional HIV incidence estimation in HIV prevention research. Journal of Acquired Immune Deficiency Syndromes 2013; 63 (Suppl. 2): S233-239.

29. Parekh BS, et al. Determination of mean recency period for estimation of HIV type 1 Incidence with the BEDcapture EIA in persons infected with diverse subtypes. AIDS Research and Human Retroviruses 2011; 27: 265-273.

30. Hauser A, et al. Improved testing of recent HIV-1 infections with the BioRad avidity assay compared to the limiting antigen avidity assay and BED Capture enzyme immunoassay: evaluation using reference sample panels from the German Seroconverter Cohort. PLoS ONE 2014; 9: e98038.

31. Longosz AF, et al. Comparison of Antibody Responses to HIV Infection in Ugandan Women Infected with HIV Subtypes A and D. AIDS Research and Human Retroviruses 2015; 31: 421-427.

32. Longosz AF, et al. Impact of HIV subtype on performance of the limiting antigen-avidity enzyme immunoassay, the bio-rad avidity assay, and the BED capture immunoassay in Rakai, Uganda. AIDS Research and Human Retroviruses 2014; 30: 339-344.

33. Longosz AF, et al. The Impact of HIV Subtype On Specificity of Cross-Sectional HIV Incidence Assays in Rakai, Uganda [Abstract]. Topics in Antiviral Medicine 2014; 22 (e-1): 532.

34. Kassanjee $\mathbf{R}$, et al. Independent assessment of candidate HIV incidence assays on specimens in the CEPHIA repository. AIDS 2014; 28: 2439-2449.

35. Yu L, et al. Low false recent rate of limiting-antigen avidity assay among long-term infected subjects from Guangxi, China. AIDS Research and Human Retroviruses. Published online: 2015. doi:10.1089/aid.2015. 0097.

36. Longosz AF, et al. Immune responses in Ugandan women infected with subtypes A and D HIV using the BED capture immunoassay and an antibody avidity assay. Journal of Acquired Immune Deficiency Syndromes 2014; 65: 390-396.

37. Laeyendecker $\mathbf{O}$, et al. Factors associated with incorrect identification of recent HIV infection using the BED capture immunoassay. AIDS Research and Human Retroviruses 2012; 28: 816-822.

38. Konikoff $\mathbf{J}$, et al. Performance of a limiting-antigen avidity enzyme immunoassay for cross-sectional estimation of HIV incidence in the United States. PLoS ONE 2013; 8: e82772.

39. Brookmeyer R, et al. Estimation of HIV incidence using multiple biomarkers. American Journal of Epidemiology 2013; 177: 264-272.

40. Longosz AF, et al. Incorrect identification of recent HIV infection in adults in the United States using a limiting-antigen avidity assay. AIDS 2014; 28: 1227 1232.

41. Laeyendecker O, et al. HIV incidence determination in the United States: a multiassay approach. Journal of Infectious Diseases 2013; 207: 232-239.

42. Eshleman SH, et al. Use of a multifaceted approach to analyze HIV incidence in a cohort study of women in the United States: HIV Prevention Trials Network 064 Study. Journal of Infectious Diseases 2013; 207: 223-31.

43. Curtis KA, et al. Evaluation of a multiplex assay for estimation of HIV-1 incidence. PLOS ONE 2013; 8: e64201.

44. Moyo S, et al. Evaluation of the false recent classification rates of multiassay algorithms in estimating HIV type 1 subtype $\mathrm{C}$ incidence. AIDS Research and Human Retroviruses 2014; 30: 29-36.

45. Hellenic Center for Disease Control and Prevention. HIV/AIDS Surveillance Report in Greece. Athens, Greece, 2014.

46. Rehle T, et al. A Comparison of South African National HIV Incidence Estimates: A Critical Appraisal of Different Methods. PLoS ONE 2015; 10: e0133255.

47. Pilcher CD, et al. Amplified transmission of HIV-1: comparison of HIV-1 concentrations in semen and blood during acute and chronic infection. AIDS 2007; 21: $1723-1730$. 
48. Blaser $\mathbf{N}$, et al. Impact of viral load and the duration of primary infection on HIV transmission: systematic review and meta-analysis. AIDS 2014; 28: 1021-1029.

49. Powers KA, et al. The role of acute and early HIV infection in the spread of HIV and implications for transmission prevention strategies in Lilongwe, Malawi: a modelling study. Lancet 2011; 378: 256-268.
50. Rottinghaus E, et al. Performance of the limitingantigen avidity EIA for use with dried blood spot specimens [Abstract]. Topics in Antiviral Medicine 2014; 22 (e-1): 531 .

51. Smith P, et al. Characterization of a rapid HIV-1 incidence assay based on a CDC-developed multisubtype antigen in a commercial HIV lateral flow assay format [Abstract]. 20th International AIDS Conference, 2014. 